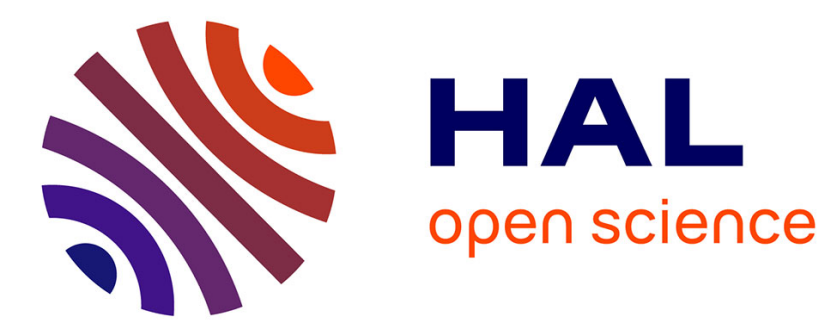

\title{
Bifurcations and symmetry breaking in the wake of axisymmetric bodies
}

David Fabre, Franck Auguste, Jacques Magnaudet

\section{To cite this version:}

David Fabre, Franck Auguste, Jacques Magnaudet. Bifurcations and symmetry breaking in the wake of axisymmetric bodies. Physics of Fluids, 2008, vol. 20, pp. 1-4. 10.1063/1.2909609 . hal-00874366

\section{HAL Id: hal-00874366 https://hal.science/hal-00874366}

Submitted on 17 Oct 2013

HAL is a multi-disciplinary open access archive for the deposit and dissemination of scientific research documents, whether they are published or not. The documents may come from teaching and research institutions in France or abroad, or from public or private research centers.
L'archive ouverte pluridisciplinaire HAL, est destinée au dépôt et à la diffusion de documents scientifiques de niveau recherche, publiés ou non, émanant des établissements d'enseignement et de recherche français ou étrangers, des laboratoires publics ou privés. 


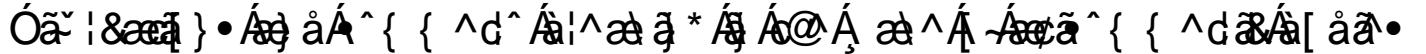

\author{
David Fabre, Franck Auguste, and Jacques Magnaudet \\ Institut de Mécanique des Fluides de Toulouse, Allée du Professeur Camille Soula, 31400 Toulouse, France
}

\begin{abstract}
We consider the generic problem of wake instabilities past fixed axisymmetric bodies, and focus on the extreme cases of a sphere and a flat disk. Numerical results reveal that the wakes of these two bodies evolve differently as the Reynolds number is increased. Especially, two new vortex shedding modes are identified behind a disk. To interpret these results, we introduce a model based on the theory of mode interactions in presence of $O(2)$ symmetry. This model, which was initially developed for the Taylor-Couette system, allows us to explain the structural differences observed in the evolution of the two types of wakes and to accurately predict the evolution of the lift force.
\end{abstract}

Physical systems that are characterized by the existence of several unstable modes are known to lead to a variety of intriguing states resulting from mode interactions. The range of possible states is especially rich in the presence of spatial symmetries. In such cases, simple nonlinear models retaining only the lowest-order nonlinear terms that respect the symmetries of the initial problem often allow most of the dynamics of the whole system to be properly captured. Normal form theory ${ }^{1,2}$ provides a systematic way to derive such models and proved to be fruitful in a wide variety of problems ranging from chemical reactions to plasmas and biological systems. We currently investigate the generic problem of the flow of a viscous incompressible fluid past fixed axisymmetric spheroidal bodies, including the extreme cases of a sphere and a flat disk. It is known that such flows spontaneously break their initial axial symmetry as the Reynolds number (defined as $R=2 U_{0} a / \nu$, where $U_{0}$ is the relative velocity between the incoming flow and the body, $a$ is the radius of the body, and $\nu$ is the kinematic viscosity of the fluid) exceeds some critical value.

In this letter, we first present computational results for two reference bodies: a sphere and a flat disk with zero thickness. While our results are essentially in agreement with available studies in the case of a sphere, ${ }^{3,4}$ they reveal two new shedding modes for a disk. We then interpret these results in the light of bifurcation theory. ${ }^{1,2}$ For this purpose, we introduce a simple model that takes into account the symmetries of the problem and describes the weakly nonlinear interaction of two unstable modes. We show that this model allows us to explain the observed structural differences between the two types of wake, as well as the discrepancies existing between numerical solutions of the full NavierStokes equations and predictions of the linear stability theory. Interestingly, this model also reveals an unexpected link between the present problem and that of the successive flow patterns in the Taylor-Couette system.

The direct numerical simulation (DNS) code and the grid used to study the flow past a sphere are described in Ref. 5. The flat disk is considered as a cylindrical obstacle with zero thickness, and the grid is strongly refined in the vicinity of its edge. It was carefully verified that the code properly resolves the corresponding flow singularity.

In the case of a sphere, a first bifurcation resulting in a loss of the axial symmetry is detected for $R \approx 210$, in agreement with both linear stability analysis ${ }^{6}$ and previous numerical studies. ${ }^{3,4}$ As displayed in Fig. 1, the resulting wake is characterized by a pair of steady streamwise vortices and exhibits a reflectional symmetry about a longitudinal plane with an arbitrary orientation. Here and in the next case, a rotation is applied to the numerical results, so that the symmetry plane coincides with the $(x, y)$ plane. A constant lift force (hence directed toward positive $y$ ) is associated with this state, which will be referred to as a steady state (SS) mode. A second bifurcation is then observed for $R \approx 270$, leading to a time-dependent flow. As shown in Fig. 2, the resulting wake retains the reflectional symmetry, but pairs of vortex structures with opposite signs are now periodically shed. Since there is still more positive (negative) vorticity on the $z<0(z>0)$ side, the average lift force remains in the positive $y$ direction but its amplitude is periodically modulated. We will refer to this state as a reflectional symmetry preserving (RSP) mode.

The present results are in good agreement with the previous numerical findings ${ }^{3,4}$ according to which the threshold for the Hopf bifurcation lies in the range of 270-274. This value slightly differs from that predicted by linear theory, ${ }^{6}$ namely, $R=277.5$. The shedding frequencies measured in DNS also differ by about $15 \%$ from those predicted by linear

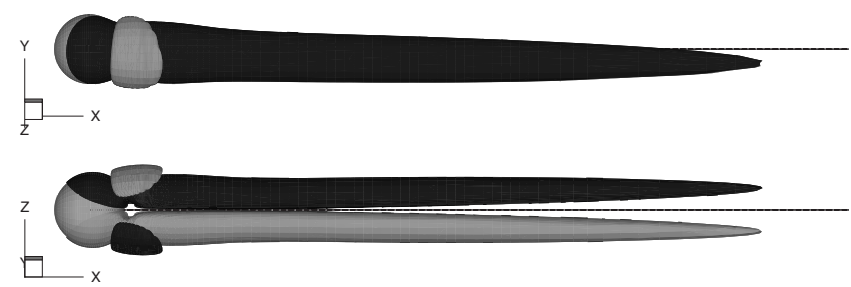

FIG. 1. SS mode in the wake of a sphere for $R=250$. The isosurfaces correspond to positive (gray) and negative (black) values of the axial vorticity. The flow comes from the $x$ direction; the thin line marks the symmetry axis of the body. 


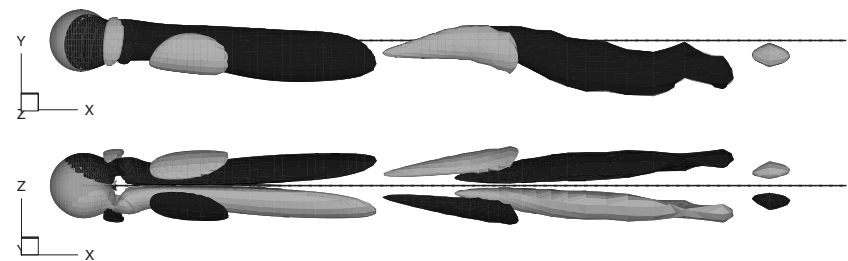

FIG. 2. RSP mode in the wake of a sphere for $R=280$.

theory. ${ }^{6}$ However, the relevance of the stability analysis of Ref. 6 is questionable as this approach is based on a linearization of the governing equations about the axisymmetric base flow which no longer exists at this point. Yet, the exact relationship between the bifurcation observed in DNS and that predicted by the linear stability theory deserves some clarification.

The case of a flat disk normal to the stream reveals a markedly different picture. We first detect a regular bifurcation for $R \approx 115$, which is in good agreement with the linear theory prediction. ${ }^{6}$ As for the sphere, this bifurcation leads to a SS mode with a reflectional symmetry. Then, a Hopf bifurcation is found for $R \approx 121$, again significantly earlier than predicted by the linear theory, ${ }^{6}$ which indicates $R=126.5$. However, the structure of the resulting flow differs from that observed in the case of the sphere, as reflectional symmetry is no longer preserved. Figure 3 shows a snapshot of the wake for $R=123$. The lift force now oscillates about a mean direction arbitrarily selected by the initial conditions. For consistency with the previous case, a rotation is applied to the numerical results so that the mean direction of the lift force coincides with the $y$ direction. This mode displays a complicated structure with vortices twisted around the symmetry axis. There is clearly no more symmetry plane but some kinds of "average" symmetry are preserved. More precisely, each half-period of the shedding cycle is the image of the other half-period through a reflection with respect to the $(x, y)$ plane. We refer to this state as a reflectional symmetry breaking (RSB) mode. Then, a third bifurcation is observed for $R \approx 140$ and allows the flow to recover the planar symmetry that was previously lost. Figure 4 depicts the wake structure for $R=150$. As can be observed, a reflexional symmetry is recovered. Interestingly, if the simulation is started from the previous case (RSB mode) and the Reynolds number is progressively increased, the symmetry plane is found to be the $(x, z)$ plane, which is orthogonal to the symmetry plane initially selected in the SS mode. In contrast with the RSP mode of Fig. 2, there is now an equal quantity of vor-

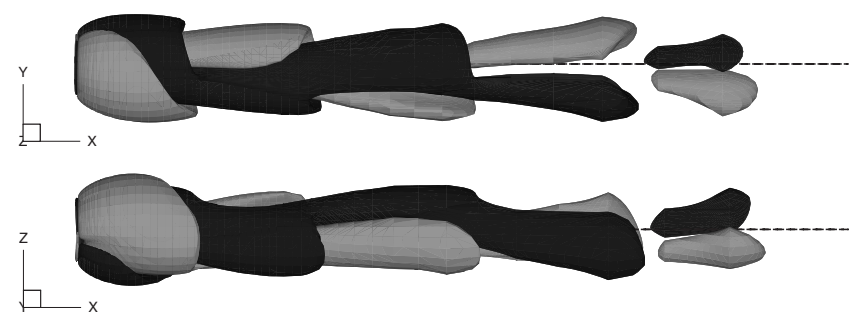

FIG. 3. RSB mode in the wake of a flat disk for $R=123$.

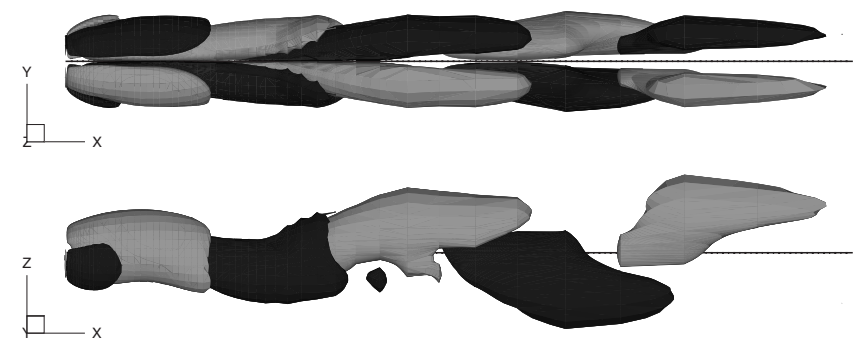

FIG. 4. SW mode in the wake of a flat disk for $R=150$.

ticity of each sign in each half-space. Consequently, the lift force (now directed along the $z$ direction) oscillates about a zero mean value. It is worth noting that this mode is more symmetrical than the ones previously described, as it displays both an exact reflectional symmetry [with respect to the $(x, z)$ plane] and an average symmetry [with respect to the $(x, y)$ plane]. For reasons explained below, we will refer to this state as a standing wave ( $\mathrm{SW}$ ) mode.

To explain the various dynamics we just described, we introduce a mathematical model based on normal form theory. We employ cylindrical coordinates $(r, \theta, x)$. Let $\mathbf{U}_{0}(r, x)$ denote the axisymmetric solution of the NavierStokes equations for a given value of $R$. Linear stability analysis of this base flow ${ }^{6}$ reveals that the dominant eigenmodes are associated with azimuthal wavenumbers $m= \pm 1$. The most amplified one is a nonoscillating mode $\hat{\mathbf{u}}_{s}(r, x)$ with a real eigenvalue $\lambda_{s}$ (obvious symmetry considerations show that the structure of this eigenmode is identical for $m=1$ and $m=-1)$. The next one is an oscillating mode $\hat{\mathbf{u}}_{h, m}(r, x)$ with a complex eigenvalue $\lambda_{h}+i \omega_{h}$, along with its conjugate counterpart. From this starting point, we consider a flow consisting of a superposition of these three modes. For this purpose, we introduce three time-dependent complex amplitudes $a_{0}, a_{1}$, and $a_{2}$ and expand the velocity field in the form

$$
\begin{aligned}
\mathbf{u}= & \mathbf{U}_{0}(r, x)+\operatorname{Re}\left[a_{0}(t) e^{-i \theta} \hat{\mathbf{u}}_{s}(r, x)\right] \\
& +\operatorname{Re}\left[a_{1}(t) e^{-i \theta} \hat{\mathbf{u}}_{h,-1}(r, x)+a_{2}(t) e^{i \theta} \hat{\mathbf{u}}_{h,+1}(r, x)\right]+\cdots .
\end{aligned}
$$

Note that the real and imaginary parts of the amplitudes can be interpreted as a measure of the intensity of the modes pointing toward the $y$ and $z$ directions, respectively.

Starting from an expansion such as Eq. (1), the central manifold theorem states that if the leading modes are simultaneously nearly neutral, the whole problem can be reduced to a system of ordinary differential equations governing their amplitudes. Moreover, this system can be reduced to its socalled normal form by taking into account the symmetries of the physical system. Note that the assumption of simultaneous nearly neutral modes is not exactly satisfied here, since the bifurcations occur at different values of $R$ (say $R_{c s}$ and $R_{c h}$ for the first two of them). However, the ratio $\left(R_{c h}-R_{c s}\right) / R_{c s}$ is fairly small, especially in the case of the disk, and the above method is known to be quite robust under such circumstances. Here, the relevant symmetry group of the problem is $O(2)$ since the base flow is invariant both 
by rotation and planar reflection. The normal form relevant to this situation is given in Ref. 1. When truncating nonlinearities at third order, it can be set into the following form:

$$
\begin{aligned}
\dot{a_{0}}= & \lambda_{s} a_{0}+l_{0}\left|a_{0}\right|^{2} a_{0}+l_{1}\left(\left|a_{1}\right|^{2}+\left|a_{2}\right|^{2}\right) a_{0} \\
& +i l_{2}\left(\left|a_{2}\right|^{2}-\left|a_{1}\right|^{2}\right) a_{0}+l_{3} \overline{a_{0} a_{2}} a_{1}, \\
\dot{a_{1}=} & \left(\lambda_{h}+i \omega_{h}\right) a_{1}+\left(B\left|a_{1}\right|^{2}+(A+B)\left|a_{2}\right|^{2}\right) a_{1} \\
& +C\left|a_{0}\right|^{2} a_{1}+D a_{0}^{2} a_{2}, \\
\dot{a_{2}=} & \left(\lambda_{h}+i \omega_{h}\right) a_{2}+\left(B\left|a_{2}\right|^{2}+(A+B)\left|a_{1}\right|^{2}\right) a_{2} \\
& +C\left|a_{0}\right|^{2} a_{2}+D{\overline{a_{0}}}^{2} a_{1} .
\end{aligned}
$$

Coefficients $l_{0}-l_{3}$ are real, while $A, B, C, D$ are complex and are written in the form $A=A_{r}+i A_{i}$, etc., in what follows. These coefficients could, in principle, be computed directly by injecting Eq. (1) into the Navier-Stokes equations and evaluating the relevant nonlinear terms. We do not attempt to follow this line here, as our aim is mainly to show that the system [Eqs. (2a)-(2c)] can qualitatively explain the observed evolutions of the wake dynamics. For simplicity, all coefficients will be considered constant (i.e., $R$-independent), and we shall assume a linear variation of the amplification rates in the form $\lambda_{s}=\alpha_{s}\left(R-R_{c s}\right), \lambda_{h}=\alpha_{h}\left(R-R_{c h}\right)$.

We are particularly concerned by the forces exerted by the fluid on the body. These forces are classically characterized by dimensionless coefficients $C_{x}, C_{y}, C_{z}$ defined as $\left(F_{x}, F_{y}, F_{z}\right)=\left(C_{x}, C_{y}, C_{z}\right) \rho \pi a^{2} U_{0}^{2} / 2$, where $\rho$ is the fluid density. From the symmetries of the flow, one may expect that the base flow $\mathbf{U}_{0}(r, x)$ only contributes to the drag force $F_{x}$, while the main contributions to the lift forces $F_{y}$ and $F_{z}$ come, respectively, from the real and imaginary parts of the mode amplitudes $a_{0}, a_{1}$, and $a_{2}$. Therefore, with an appropriate rescaling of the eigenmodes, the leading-order contributions to the lift coefficients can be expanded in the form

$$
C_{y}=a_{0, r}+a_{1, r}+a_{2, r}+\cdots, \quad C_{z}=a_{0, i}+a_{1, i}-a_{2, i}+\cdots .
$$

A mathematical exploration of the solutions of Eqs. (2a)-(2c), up to ternary bifurcations, was performed in Ref. 1 . Here, we only describe the solutions relevant to our problem. The simplest solution of Eqs. (2a)-(2c) is the SS mode which bifurcates when $\lambda_{s}>0$. This bifurcation is governed by Eq. (2a) with only the first nonlinear term kept. Setting $a_{0}=r_{0} e^{i \phi_{0}}$, the saturated amplitude is $r_{0}=\sqrt{-\lambda_{s} / l_{0}}$, thus corresponding to a regular supercritical bifurcation if $l_{0}<0$. The above truncation accurately describes the occurrence of the lift force observed with both bodies for $R>R_{c s}$. Note that the phase $\phi_{0}$, which corresponds to the direction of the lift force, is arbitrary, i.e., selected by initial disturbances.

For $\lambda_{h}>0$, the normal form of Eqs. (2a)-(2c) predicts that a primary Hopf bifurcation occurs on the axisymmetric solution. This bifurcation is not observed in the present cases because the axisymmetric state has already lost its stability, owing to the regular bifurcation we just described. Nevertheless, it is useful to discuss this bifurcation first in order to derive a consistent bifurcation diagram. Because of the $O(2)$ symmetry properties, ${ }^{1,2}$ this bifurcation gives rise to two branches, classically called rotating waves (RW) (with $a_{2}=0$ or $a_{1}=0$ ) and SW (with $\left|a_{2}\right|=\left|a_{1}\right|$ ). The RW mode corresponds to a state where the lift force has a constant amplitude and a rotating orientation, and such a state is not observed. On the other hand, the SW solution is relevant to the mode observed in the wake of a flat disk after the third bifurcation. Introducing the polar representation $a_{1}=r_{S} e^{i \phi_{1}}$, $a_{2}=r_{S} e^{i \phi_{2}}$, the saturated characteristics of the SW mode are given by $r_{S}=\sqrt{-\lambda_{h} /\left(2 B_{r}+A_{r}\right)}$, and $\dot{\phi}_{1}=\dot{\phi}_{2}=\omega_{h}-\left(2 B_{i}\right.$ $\left.+A_{i}\right) \lambda_{h} /\left(2 B_{r}+A_{r}\right)$. Using Eq. (3), it can be checked that the corresponding lift force is oscillating about zero in a fixed plane, just as the mode described in Fig. 4. According to Refs. 1 and 2, the bifurcation toward the RW (SW) branch is supercritical if $B_{r}<0\left(2 B_{r}+A_{r}<0\right)$; if both conditions are satisfied, the bifurcation preferentially leads to the SW solution if $A_{r}>0$. We assume all these conditions to be satisfied here.

Two Hopf bifurcations can arise on the SS branch, giving rise to two secondary branches ${ }^{1}$ which, by considering their symmetry properties, can be identified with the RSP and RSB states described above. Some conditions involving the parameters of the normal form determine which branch is preferentially selected. ${ }^{1}$ With present notations, it turns out that the RSP (RSB) mode is selected if $D_{r}>0\left(D_{r}<0\right)$. The threshold $R_{c}^{*}$ corresponding to this secondary bifurcation as well as the marginal frequency $\omega^{*}$ of the resulting mode can be obtained by linearizing the normal form about the SS solution. Assuming linear variations of $\lambda_{s}$ and $\lambda_{h}$ as specified above, it is found that $R_{c}^{*}=R_{c h}-\alpha_{s}\left(C_{r} \pm D_{r}\right)\left(R_{c h}\right.$ $\left.-R_{c s}\right) /\left[\alpha_{s}\left(C_{r} \pm D_{r}\right)-l_{0} \alpha_{h}\right], \quad$ and $\quad \omega^{*}=\omega_{h}-\left(C_{i} \pm D_{i}\right) \alpha_{s}\left(R_{c}^{*}\right.$ $\left.-R_{c h}\right) / l_{0}$, respectively, where the sign has to be taken as positive (negative) for the bifurcation toward the RSP (RSB) branch. These expressions provide a simple explanation of the aforementioned differences between the predictions from linear stability theory and full Navier-Stokes computations on both the critical Reynolds number and the frequency of the secondary bifurcation. Finally, the third bifurcation observed for the disk can be similarly explained as a (backward) secondary bifurcation from the SW state to the RSB state. With the present notation, it is found that such a bifurcation requires $l_{3}<0^{1}$.

Interestingly, the normal form of Eqs. (2a)-(2c) is also relevant to another classical flow configuration, namely the Taylor-Couette flow. The mathematical study performed in Ref. 1 was initially motivated by this application. In this case, the $O(2)$ symmetry results from the hypothesis of reflectional symmetry and periodicity of the flow along the axial direction. Such a symmetry is approximate and is only exact in the limit of an infinitely long device. Considering their symmetry properties, the present SS, RW, SW, RPM, and RBM states can be identified with the "Taylor vortices," "spirals," "ribbons," "twisted vortices," and "wavy vortices" flow patterns, respectively.

From the above considerations, we are in a position to build consistent bifurcation diagrams for the flow past a sphere and a disk. Such diagrams are displayed in Fig. 5. The 

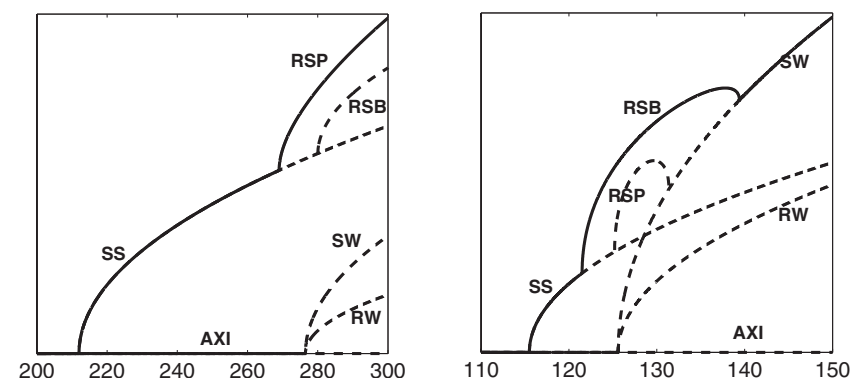

FIG. 5. Theoretical bifurcation diagrams for the sphere (left) and the flat disk (right). Solid (dashed) lines denote stable (unstable) branches.

left plot corresponds to the case of the sphere with $D_{r}>0$. In this case, a secondary bifurcation occurs on the SS branch, giving birth to a stable RSP branch. A RSB branch can also be present but is necessarily unstable. The right plot corresponds to the case of the disk with $D_{r}<0$ and $l_{3}<0$. Here, a stable RSB branch exists within a limited interval of $R$ and links the SS and SW branches (a RSP branch also exists in this case but is unstable).

The assumption of simultaneous nearly neutral modes is most closely satisfied for the disk, making it quite easy to determine a set of parameters providing a quantitative fitting of the numerical results. The linear growth rates were estimated from observations of the growth or decay of small amplitude disturbances in the vicinity of the thresholds. The linear interpolation procedure yielded $\lambda_{s}=0.0053(R-115.5)$ and $\lambda_{h}=0.0045(R-126.5)$. The other parameters were determined through a best fit procedure, leading to $\omega_{h}=1.49$, $l_{0}=-14.6, l_{1}=-750, l_{2}=0, l_{3}=-160, B=-340, A=340, C=3$, and $D=-2.7$. With this set of parameters, the thresholds for the two primary bifurcations agree with the predictions of linear stability theory. ${ }^{6}$ Then, the RSB branch is found to bifurcate from the SS branch at $R=R_{c}^{*}=121.5$, and to end on the SW branch for $R=139.6$, which is in good agreement with the DNS results. Figure 6 compares the lift coefficients obtained with this set of parameters to the DNS results (note that we plot the mean value of $C_{y}$ which exhibits small oscillations in the RSB mode, and the maxima of $C_{z}$, which oscillate about a zero mean in the SW mode). As can be seen, the essential features of the numerical results are captured by the model. The largest departures occur in the range of Reynolds numbers where the amplitudes are the largest and the truncation to third order is most questionable.

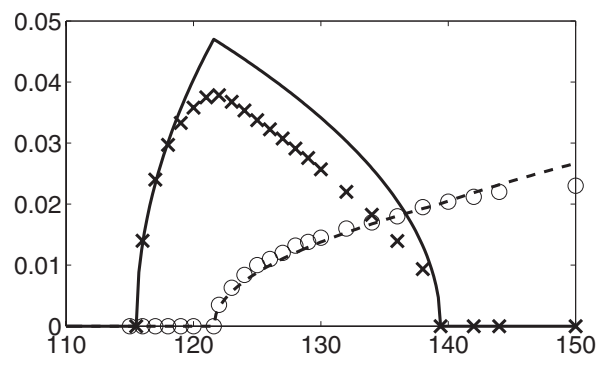

FIG. 6. Lift coefficients of a flat disk vs $R$. Solid line (crosses): predictions (DNS results) for $C_{y}$. Dashed line (open circles): predictions (DNS results) for the maxima of $C_{z}$.

The normal form introduced here has thus proved to be successful to explain the observed differences in the wakes of a fixed disk and a fixed sphere. This approach is also applicable to the wakes of axisymmetric bodies of any particular shape, and opens new ways to explore such problems. In particular, applying the present theory to moving bodies could be of great interest, the most challenging situation being that of freely moving bodies whose motion is driven by buoyancy, such as rising bubbles. The path of such bodies is known to exhibit a variety of forms, including planar zigzags, helices, tumbling motions, etc. It is now recognized ${ }^{5,7}$ that such path instabilities are directly linked to an instability of the recirculating region in the near wake of the body. Extending the present approach to such problems, in which the geometrical degrees of freedom of the body are intimately coupled to the wake dynamics, is an extremely challenging question to which we will devote future efforts.

We thank Edgar Knobloch for stimulating discussions.

${ }^{1}$ M. Golubitsky, I. Stewart, and D. G. Schaeffer, Singularities and Groups in Bifurcation Theory, Applied Mathematical Sciences Vol. II (Springer, New York, 1988).

${ }^{2}$ J. D. Crawford and E. Knobloch, "Symmetry and symmetry-breaking bifurcations in fluid dynamics," Annu. Rev. Fluid Mech. 23, 341 (1991).

${ }^{3}$ T. A. Johnson and V. C. Patel, "Flow past a sphere up to Reynolds number of 300," J. Fluid Mech. 378, 19 (1999).

${ }^{4}$ A. G. Tomboulides and S. A. Orzag, "Numerical investigation of transitional and weak tyurbulent flow past a sphere," J. Fluid Mech. 416, 45 (2000).

${ }^{5}$ J. Magnaudet and G. Mougin, "Wake instability of a fixed spheroidal bubble," J. Fluid Mech. 572, 311 (2007).

${ }^{6}$ R. Natarajan and A. Acrivos, "The instability of the steady flow past sphere and disks," J. Fluid Mech. 254, 323 (1993).

${ }^{7}$ G. Mougin and J. Magnaudet, "Path instability of a rising bubble," Phys. Rev. Lett. 88, 014502 (2002). 